\title{
Undercarboxylated osteocalcin correlates with insulin secretion in Japanese individuals with diabetes
}

\author{
Shogo Funakoshi', Kumiko Yoshimura 1,2, Seiki Hirano' , Satoko Ohmi', Eri Amano', Yoshiharu Fukuda²,
} Yoshio Terada ${ }^{1}$ and Shimpei Fujimoto ${ }^{1 *}$ (D)

\begin{abstract}
Background: Undercarboxylated osteocalcin (UCOC) is a secreted protein produced by osteoblasts that regulates insulin secretion and insulin sensitivity in rodents. However, the significance of these effects on glucose metabolism in human remains unknown. Moreover, the pathophysiological roles of ucOC on varying degrees of glucose intolerance, including diabetes need to be elucidated. In the present study, correlations between ucOC and indices of insulin secretion and sensitivity were analyzed in normal glucose tolerance (NGT), impaired glucose metabolism (IGM), and diabetes mellitus (DM) groups.
\end{abstract}

Methods: Based on $75 \mathrm{~g}$ OGTT data in Japanese individuals without diabetic medication, or medications which may affect ucOC levels, individuals were classified as having normal glucose tolerance (NGT), impaired glucose metabolism (IGM), or diabetes (DM). In each group, 25 individuals were consecutively recruited [total 75 individuals, age: $65 \pm 11$ (mean \pm SD); BMI: $24.9 \pm 3.8 \mathrm{~kg} / \mathrm{m}^{2}$ ]. QUICKI and Matsuda index (MI) were calculated as insulin sensitivity indices. Homeostasis model assessment (HOMA)- $\beta$ and insulinogenic index (IGI) were calculated as insulin secretion indices. UcOC was measured using ECLIA. Normally-distributed $\log _{\mathrm{e}}$-transformed (In-) values were used for ucOC, HOMA- $\beta, I G I$, and MI.

Results: The ucOC was not significantly different among the three groups. The results of multiple regression analysis showed that In-ucOC did not significantly correlate with age, sex, BMI, waist circumference, fasting plasma glucose, plasma glucose 120 min after glucose loading, fasting plasma immunoreactive insulin, In-HOMA- $\beta$, QUICKI, or In-MI in any of the three groups. Interestingly, In-ucOC correlated with In-IGI $(r=0.422, P=0.0354)$ and HbA1c $(r=-0.574$, $P=0.0027)$ only in the DM group. There was no significant correlation between In-IGI and age, sex, BMI, or HbA1c in the DM group. Further, the results of multiple regression analysis showed that In-IGI could be independently predicted by BMI $(\beta=0.598, P=0.0014)$ and In-uCOC $(\beta=0.641, P=0.0007)$ in the DM group $\left(R^{2}=0.488, P=0.0006\right)$.

Conclusion: In our study, ucOC positively correlated with insulin secretion independently of BMI in Japanese individuals with diabetes. These results suggest that $\mathrm{UCOC}$ plays more important roles in insulin secretion than in insulin sensitivity in individuals with diabetes.

Keywords: Insulin secretion, BMI, Undercarboxylated osteocalcin

\footnotetext{
${ }^{*}$ Correspondence: fujimoto@kochi-u.ac.jp

${ }^{1}$ Department of Endocrinology, Metabolism, and Nephrology, Kochi

Medical School, Kochi University, Kohasu, Oko-cho, Nankoku, Kochi 783-8505, Japan

Full list of author information is available at the end of the article
}

\section{Background}

In recent years, evidences from studies on mice have suggested that bone is a key organ that modulates glucose metabolism [1-3]. Osteocalcin $(\mathrm{OCN})$, a known 
biomarker of bone turnover which is a unique protein synthesized by osteoblasts [2], plays important roles in glucose metabolism, since OCN-deleted mice exhibited hypoinsulinemia, hyperglycemia, glucose intolerance and insulin resistance [1].

In murine studies, these function of $\mathrm{OCN}$ are fulfilled by its undercarboxylated form, namely undercarboxylated osteocalcin (ucOC) [2]. G protein-coupled receptor C6A (GPRC6A), found in the Leydig cells of the testis, is also expressed in pancreatic $\beta$-cells [4]. In mouse $\beta$ cells, ucOC promotes proliferation via GPRC6A and increases insulin secretion $[2,4]$. UcOC also promotes $\beta$ cell proliferation and insulin secretion in human pancreatic islets [5]. Osteocalcin exerts a favorable influence on insulin sensitivity in mice through a poorly understood mechanism $[1,2]$. Recently, it is revealed that OCN signaling via GPRC6A favors glucose uptake and utilization by myofibers [6].

In cross-sectional studies, higher serum levels of OCN were negatively related to fasting plasma glucose (FPG) and glycated hemoglobin A1c (HbA1c) in individuals with mainly normal and impaired glucose tolerance [7-9]. Although basic studies have indicated that ucOC affects both insulin secretion and insulin sensitivity, the significance of these effects on glucose metabolism in human remains unknown. Moreover, the pathophysiological roles of ucOC on varying degrees of glucose intolerance, including diabetes need to be elucidated. In the present study, correlations between ucOC and indices of insulin secretion and sensitivity were analyzed in normal glucose tolerance (NGT), impaired glucose metabolism (IGM), and diabetes mellitus (DM) groups.

\section{Methods}

\section{Participants}

We analyzed data derived from a $75 \mathrm{~g}$ oral glucose tolerance test (75gOGTT) involving 75 Japanese individuals who did not take any diabetic medication $[\operatorname{sex}(\mathrm{M} / \mathrm{F})$ : 34/41; age: $65 \pm 11$ (mean \pm SD); BMI: $\left.24.9 \pm 3.8 \mathrm{~kg} / \mathrm{m}^{2}\right]$, as described previously [10]. Participants did not take warfarin and any anti-osteoporosis medication including vitamin $\mathrm{D}$, vitamin $\mathrm{K}$, and bisphosphonate. The study protocol was approved by the Ethical Review Board of Kochi Medical School, Japan.

\section{Laboratory examinations and anthropometric data}

Based on 75gOGTT data, individuals were either classified as NGT, IGM, or DM according to the 2006 World Health Organization (WHO) criteria [11] [NGT, fasting plasma glucose (FPG) $<110 \mathrm{mg} / \mathrm{dL}$ and 2 -h plasma glucose (2-h PG) $<140 \mathrm{mg} / \mathrm{dL}$; IGM, either impaired fasting glucose (IFG, FPG $\geq 110$ and $<126 \mathrm{mg} / \mathrm{dL}$ ) and/or impaired glucose tolerance (IGT, $2-h$ PG $\geq 140$ and $<200 \mathrm{mg} / \mathrm{dL}$ ); DM, FPG $\geq 126 \mathrm{mg} / \mathrm{dL}$ and/or 2 -h $\mathrm{PG} \geq 200 \mathrm{mg} / \mathrm{dL}$ ].Fasting serum levels of ucOC were measured using a ucOC electro-chemiluminescence immunoassay (ECLIA) Kit (Picolumi ucOC, Eidia Co., Ltd., Tokyo). Although fasting plasma glucose, plasma glucose 120 min after glucose load, and HbA1c were significantly different among the 3 groups, age, sex, BMI were not significantly different as previously described [10].

\section{Indices of insulin secretion and insulin sensitivity}

Indices of insulin secretion [homeostasis model assessment (HOMA) $\beta$ and insulinogenic index (IGI)] and insulin sensitivity [Quantitative Insulin Sensitivity Check Index (QUICKI) and the Matsuda index (MI)] were calculated using glucose and immunoreactive insulin (IRI) data from the $75 \mathrm{~g}$ OGTT, as previously described [12]. As indices of insulin secretion, the ratio of the areaunder-the-insulin-curve to the area-under-the-glucosecurve during $0-120 \mathrm{~min}$ of the OGTT $\left(\mathrm{AUC}_{\text {ins/gluc }}\right)$, which reflects glucose-induced insulin secretion in not only early phase but also in late phase [13] and the insulin secretion-sensitivity index-2 (ISSI-2) [14, 15], an oral disposition index that reflects the compensatory insulin secretion capacity against decreased insulin sensitivity (increased insulin resistance), were also calculated. To calculate ISSI-2, the $\mathrm{AUC}_{\text {ins/gluc }}$ was multiplied by the MI. The IGI, QUICKI, and MI, but not HOMA- $\beta$, have been shown to be significantly different among the NGT, IGM, and DM groups [10].

\section{Statistical analysis}

Normally distributed continuous data are presented as mean $\pm \mathrm{SD}$ and non-normally distributed continuous data are presented as median value, 25th percentile value and 75 th percentile value. Difference among more than three groups was determined by analysis of variance (ANOVA) for normally distributed continuous data, by Kruskal-Wallis tests for non-normally distributed continuous data, and Scheffe's test was performed as post hoc analysis. The relationship between the parametric data was determined using the Pearson's correlation coefficients analysis. In regression analyses, normally distributed $\log _{\mathrm{e}}$-transformed $(\mathrm{ln}-)$ values were performed

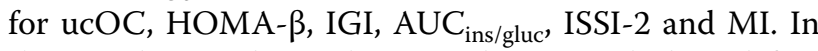
the simple correlation between $\ln$-IGI and clinical factors including age, sex (female $=0$, male $=1), B M I$, and HbA1c in the DM group, the BMI coefficient ( $r$ ) was highest ( $\mathrm{r}$ of age, sex, BMI, and HbA1c were -0.069 , $0.022,0.363$, and 0.011 , respectively). Therefore, in the multiple regression analysis of the DM group, in which ln-IGI was a dependent variable and $\ln$-ucOC was an independent variable, BMI was selected as an additional 
independent variable. $\mathrm{P}$ values $<0.05$ were considered statistically significant.

\section{Results}

\section{Biochemical profiles of groups according to glucose} tolerance

The ucOC was not significantly different among the three groups (Table 1). Indices of insulin secretion including $\mathrm{AUC}_{\text {ins/gluc }}$ and ISSI-2 differed among the NGT, IGM, and DM groups (Table 1).

\section{Simple regression analyses}

Ln-ucOC did not significantly correlate with age, sex, BMI, waist circumference, fasting plasma glucose, plasma glucose $120 \mathrm{~min}$ after glucose loading, fasting plasma immunoreactive insulin, ln-HOMA- $\beta$, QUICKI, or $\ln -\mathrm{MI}$ in any of the three groups (Table 2). Interestingly, ln-ucOC correlated with $\ln$-IGI $(\mathrm{r}=0.422$, $P=0.0354), \ln -\mathrm{AUC}_{\text {ins/gluc }}(\mathrm{r}=0.397, P=0.0491), \mathrm{ln}-$ ISSI-2 $(\mathrm{r}=0.536, P=0.0057)$ and HbA1c $(\mathrm{r}=-0.574$, $P=0.0027$ ) only in the DM group (Table 2, Fig. 1).

\section{Multiple regression analysis in the DM group}

Ln-IGI was independently predicted by BMI $(\beta=0.598$, $\mathrm{P}=0.0014) \quad$ and $\ln -\mathrm{ucOC} \quad(\beta=0.641, \quad \mathrm{P}=0.0007)$ $\left(\mathrm{R}^{2}=0.488,|\mathrm{R}|=0.699, P=0.0006\right)$.

Table 1 The serum ucOC levels and insulin secretion indices in the NGT, IGM and DM groups

\begin{tabular}{lllll}
\hline & NGT $(\mathbf{n}=\mathbf{2 5})$ & IGM $(\mathbf{n}=\mathbf{2 5})$ & DM $(\mathbf{n}=\mathbf{2 5})$ & P \\
\hline ucOC $(\mathrm{ng} / \mathrm{mL})$ & $4.3(3.1,5.2)$ & $4.0(3.3,6.6)$ & $4.0(2.6,5.8)$ & 0.9411 \\
Ln-uCOC & $1.407 \pm 0.516$ & $1.383 \pm 0.817$ & $1.389 \pm 0.606$ & 0.9914 \\
AUC $_{\text {ins/gluc }}(\mathrm{pmol} / \mathrm{mmol})$ & $29.7(20.4,51.4)$ & $32.4(19.2,46.0)$ & $22.0(13.3,31.7)$ & 0.0295 \\
Ln- AUC $_{\text {ins/gluc }}$ & $3.452 \pm 0.593$ & $3.465 \pm 0.573$ & $3.046 \pm 0.545^{*, * * *}$ & 0.0164 \\
ISSI-2 & $181.9(164.8,219.5)$ & $119.8^{*}(103.3,158.3)$ & $77.7^{*, * *}(59.4,85.9)$ & $<0.0001$ \\
Ln-ISSI-2 & $5.266 \pm 0.314$ & $4.869 \pm 0.351^{*}$ & $4.286 \pm 0.334^{*, * *}$ & $<0.0001$ \\
\hline
\end{tabular}

Median values are shown. The 25 th percentile and 75 th percentile values are shown in parenthesis

ucOC undercarboxylated osteocalcin, $A \cup C_{\text {ins/gluc }}$ the ratio of the area-under-the-insulin-curve to the area-under-the-glucose-curve, ISS1-2 the component measures of the insulin secretion-sensitivity index-2

* $\mathrm{P}<0.01$ vs NGT; ${ }^{*} \mathrm{P}<0.01$ vs IGM

Table 2 The simple correlation between In-UcOC and clinical factors in the NGT, IGM, and DM groups

\begin{tabular}{|c|c|c|c|c|c|c|}
\hline & \multicolumn{2}{|c|}{ NGT $(n=25)$} & \multicolumn{2}{|c|}{ IGM $(n=25)$} & \multicolumn{2}{|c|}{$D M(n=25)$} \\
\hline & $r$ & $P$ & $r$ & $P$ & $r$ & $P$ \\
\hline Age & -0.170 & 0.4174 & 0.280 & 0.1857 & 0.268 & 0.1959 \\
\hline $\operatorname{Sex}(F=0, M=1)$ & -0.381 & 0.0606 & -0.141 & 0.5123 & -0.241 & 0.2456 \\
\hline BMl & 0.395 & 0.0505 & 0.006 & 0.9783 & -0.366 & 0.0719 \\
\hline WC & 0.295 & 0.1527 & -0.019 & 0.9315 & -0.136 & 0.5175 \\
\hline $\mathrm{HbA1c}(\%)$ & -0.048 & 0.8199 & 0.239 & 0.2608 & -0.574 & 0.0027 \\
\hline $\mathrm{G}_{0}$ & 0.275 & 0.1834 & 0.307 & 0.1444 & -0.392 & 0.0525 \\
\hline $\mathrm{G}_{120}$ & 0.302 & 0.1422 & 0.123 & 0.5662 & -0.222 & 0.2867 \\
\hline $\mathrm{I}_{0}$ & 0.138 & 0.5109 & -0.231 & 0.2773 & -0.249 & 0.2291 \\
\hline In-HOMA- $\beta$ & 0.089 & 0.6722 & -0.332 & 0.1127 & 0.177 & 0.3961 \\
\hline$|n-| G \mid$ & 0.040 & 0.8487 & -0.373 & 0.0726 & 0.422 & 0.0354 \\
\hline In-AUC ins/gluc & 0.128 & 0.5406 & -0.281 & 0.1838 & 0.397 & 0.0491 \\
\hline In-ISSI-2 & -0.233 & 0.2619 & -0.298 & 0.1577 & 0.536 & 0.0057 \\
\hline QUICKI & -0.280 & 0.1756 & 0.082 & 0.7047 & 0.151 & 0.4718 \\
\hline$|n-M|$ & -0.264 & 0.2024 & 0.108 & 0.6155 & -0.069 & 0.7434 \\
\hline
\end{tabular}

$\mathrm{G}_{0}$, and $\mathrm{G}_{120}$ indicate plasma glucose at 0 (fasting) and 120 min after glucose loading in $75 \mathrm{gOGTT}$, respectively. $\mathrm{I}_{0}$ is plasma immunoreactive insulin (IRI) at 0 (fasting) $B M I$ body mass index, WC waist circumference, QUICKI Quantitative Insulin Sensitivity Check Index, HOMA homeostasis model assessment, IGI insulinogenic index, MI Matsuda index 


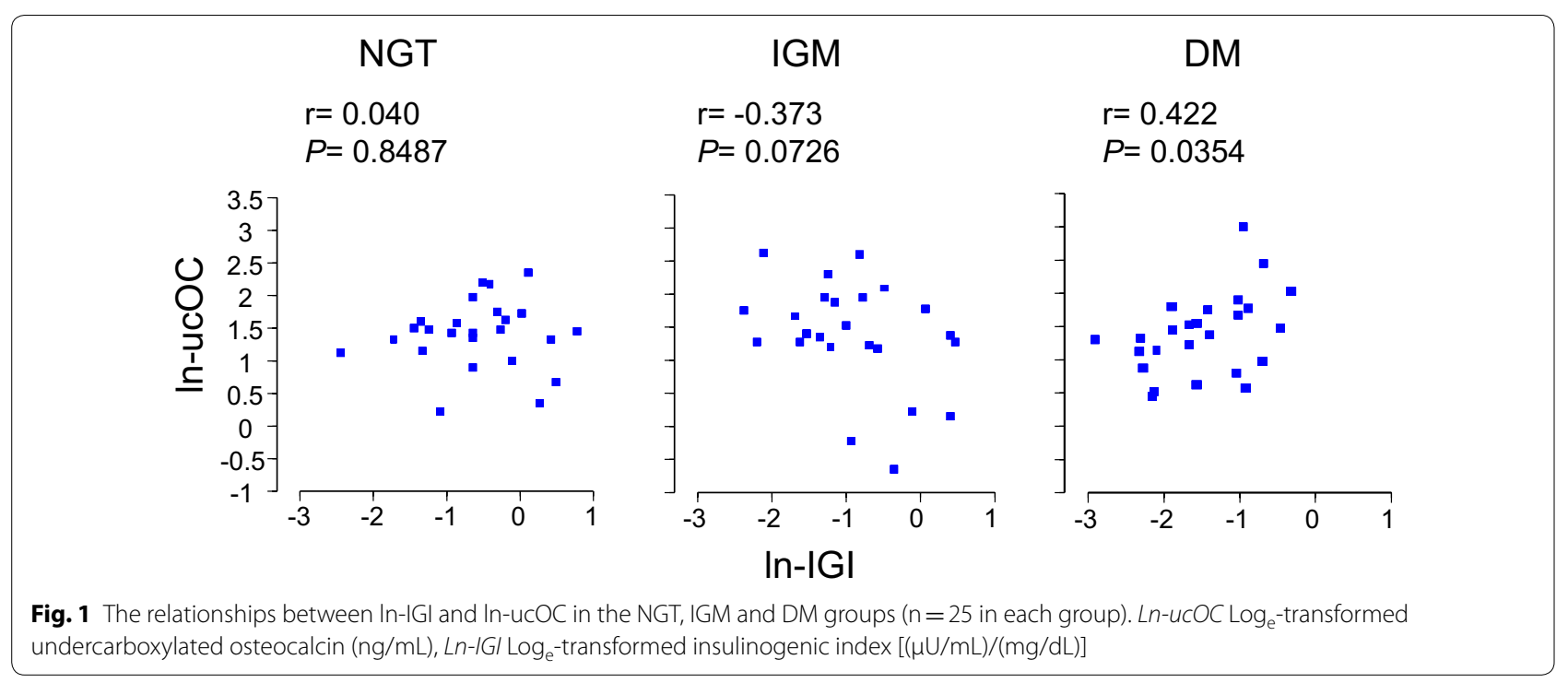

\section{Discussion}

In the previous cross-sectional human studies, the 75gOGTT was not performed and insulin secretion was evaluated checking HOMA- $\beta$ levels [7-9], and not by IGI. In a Japanese population, AUC for receiver operating characteristic (ROC) to predict the incidence of diabetes of IGI (0.825) is far larger than that of HOMA- $\beta$ (0.604) [16]. Before the incidence of diabetes in women with gestational diabetes mellitus, IGI, but not HOMA- $\beta$, of individuals who had developed to diabetes is significantly lower than that of individuals who had not developed to diabetes [17]. Moreover, the utility of HOMA- $\beta$ to predict diabetes development is questioned in nondiabetic Koreans [18]. These results indicate that IGI is a more reliable index of insulin secretion than HOMA- $\beta$ in pathophysiological aspects. In addition, there are no previous reports showing the association between ucOC and glucose metabolism among groups classified into NGT, IGM, and DM groups with matched clinical backgrounds.

In this study, basal insulin secretion was not correlated with ucOC in diabetes as shown previously $[19,20]$. However, we showed for the first time that serum ucOC is positively correlated with IGI, which reflects insulin secretion in early phase, in individuals with diabetes. Previous studies indicated that BMI was positively correlated with insulin secretion in individuals with diabetes, which might reflect compensatory augmentation of insulin secretion against insulin resistance [21,22]. The present study also shows that ucOC correlates with IGI independent of BMI, thereby indicating it to be a novel factor to correlate with insulin secretion in individuals with diabetes. In addition, ucOC correlated with $\mathrm{AUC}_{\text {ins/gluc }}$ and
ISSI-2, and this finding reflects the association between ucOC and insulin secretion in the early and late phases and accounts for the compensatory capacity against insulin resistance.

Interestingly, our results showed that $\mathrm{ucOC}$ was negatively correlated with $\mathrm{HbA1c}$ in the DM group. Since IGI did not correlate with HbA1c in the DM group in the present study, the correlation between ucOC and HbA1c via insulin secretion seems unlikely. In a previous study, chronic exposure to high concentrations of glucose showed a decrease in OCN gene transcription in osteoblast-like cell lines [23]. It can therefore be postulated that the suppressive effect on ucOC from osteoblasts, due to chronic exposure to high glucose, may play a role in impaired glucose-induced insulin secretion in diabetes.

Although basic studies demonstrated that ucOC affects insulin sensitivity, it did not correlate with indices of insulin sensitivity in any of the three groups in the present study (Table 2). In many literatures, total OCN including carboxylated $\mathrm{OCN}$ is weakly associated with insulin resistance $[7-9,24]$ for unknown reasons. The present results are compatible with separately shown results in which ucOC does not associate with insulin resistance in individuals with diabetes [20] and without diabetes [24].

\section{Limitations}

The present study has limitations. First, since it was a cross-sectional study, a causal association could not be evaluated. It is unclear why the correlation between ucOC and insulin secretion was found only in individuals with diabetes. Second, since data were collected at a 
single institute in Japan, this study may have suffered a selection bias and therefore may not represent the broader population. Third, since the sample number is not large enough, weak correlations might be regarded as not significant and the influence of sex, which may affect ucOC levels, on the association between ucOC and insulin secretion was not analyzed in this study. Fourth, we did not measure plasma vitamin K levels. Prominent vitamin $\mathrm{K}$ deficiency is rare in the Japanese population except for patients with nutritional disorders and the individuals who were included in the present study did not have nutritional disorders and did not take warfarin. Since plasma vitamin $\mathrm{K}$ weakly and negatively correlates with ucOC in healthy Japanese women [25], vitamin K may also affect ucOC in IGM and DM. The influence of vitamin $\mathrm{K}$ on the association between ucOC and insulin secretion remains unknown.

\section{Conclusion}

In conclusion, in our study, ucOC positively correlated with insulin secretion independently of BMI in Japanese individuals with diabetes. This suggests that in humans, ucOC may play more important roles in insulin secretion than in insulin sensitivity in individuals with diabetes.

\begin{abstract}
Abbreviations
OGTT: Oral glucose tolerance test; IRI: Immunoreactive insulin; IGI: Insulinogenic index; HOMA: Homeostasis model assessment; QUICKI: Quantitative insulin sensitivity check index; $M I$ : Matsuda index; $A \cup C_{\text {ins/gluc: }}$ The ratio of the area-under-the-insulin-curve to the area-under-the-glucose-curve during 0-120 min of the OGTT; ISSI-2: Insulin secretion-sensitivity index-2; UCOC: Undercarboxylated osteocalcin; ECLIA: Electro-chemiluminescence immunoassay; HbA1c: Glycated hemoglobin; NGT: Normal glucose tolerance; IGM: Impaired glucose metabolism; DM: Diabetes mellitus; G: Glucose; BMI: Body mass index.
\end{abstract}

\section{Acknowledgements}

The authors thank Ms. Reiko Matsumoto from the Department of Endocrinology, Metabolism, and Nephrology, Kochi Medical School, Kochi University for technical assistance.

\section{Authors' contributions}

SFunakoshi, YT and SFujimoto participated in the design of study. KY, SH, SO, EA, and YF participated in data collection. SFunakoshi and SFujimoto participated in analysis of data and wrote the manuscript. All authors read and approved the final manuscript.

\section{Funding}

The authors have received no funding support regarding this study.

\section{Availability of data and materials}

The ethics committee imposed restrictions to data access and sharing. Individuals who wish to access our data must obtain further permission from the committee, which can be achieved by contacting the corresponding author.

\section{Ethics approval and consent to participate}

The study protocol was approved by the Ethical Review Board of Kochi Medical School. Informed consent was obtained from each patient.

\section{Consent for publication}

Not applicable.

\section{Competing interests}

None of the authors have any competing interests.

\section{Author details}

${ }^{1}$ Department of Endocrinology, Metabolism, and Nephrology, Kochi Medical School, Kochi University, Kohasu, Oko-cho, Nankoku, Kochi 783-8505, Japan.

${ }^{2}$ Fukuda Clinic, Kochi, Kochi 780-0023, Japan.

Received: 22 May 2020 Accepted: 12 August 2020

Published online: 17 August 2020

\section{References}

1. Lee NK, Sowa H, Hinoi E, Ferron M, Ahn JD, Confavreux C, et al. Endocrine regulation of energy metabolism by the skeleton. Cell. 2007;130:456-69.

2. Liu JM, Rosen CJ, Ducy P, Kousteni S, Karsenty G. Regulation of glucose handling by the skeleton: insights from mouse and human studies. Diabetes. 2016;65:3225-322.

3. Liu TT, Liu DM, Xuan Y, Zhao L, Sun LH, Zhao DD, et al. The association between the baseline bone resorption marker CTX and incident dysglycemia after 4 years. Bone Res. 2017;5:17020.

4. Wei J, Hanna T, Suda N, Karsenty G, Ducy P. Osteocalcin promotes $\beta$-cell proliferation during development and adulthood through Gprc6a. Diabetes. 2014;63:1021-31.

5. Sabek OM, Nishimoto SK, Fraga D, Tejpal N, Ricordi C, Gaber AO. Osteocalcin effect on human $\beta$-cells mass and function. Endocrinology. 2015;156:3137-46.

6. Mera P, Laue K, Ferron M, Confavreux C, Wei J, Galán-Díez M, et al. Osteocalcin signaling in myofibers is necessary and sufficient for optimum adaptation to exercise. Cell Metab. 2016;23:1078-92.

7. Bae SJ, Choe JW, Chung YE, Kim BJ, Lee SH, Kim HY, et al. The association between serum osteocalcin levels and metabolic syndrome in Koreans. Osteoporos Int. 2011;22:2837-46.

8. Bao Y, Zhou M, Lu Z, Li H, Wang Y, Sun L, et al. Serum levels of osteocalcin are inversely associated with the metabolic syndrome and the severity of coronary artery disease in Chinese men. Clin Endocrinol (Oxf). 2011;75:196-201.

9. Dou J, Ma X, Fang Q, Hao Y, Yang R, Wang F, et al. Relationship between serum osteocalcin levels and non-alcoholic fatty liver disease in Chinese men. Clin Exp Pharmacol Physiol. 2013;40:282-8.

10. Yoshimura K, Hirano S, Takata H, Funakoshi S, Ohmi S, Amano E, et al. Plasma mannose level, a putative indicator of glycogenolysis, and glucose tolerance in Japanese individuals. J Diabetes Investig. 2017:8:489-95

11. World Health Organization. Definition and diagnosis of diabetes mellitus and intermediate hyperglycemia: report of a WHO/IDF consultation. Geneva: World Health Organization; 2006.

12. Ohmi S, Ono M, Takata H, Hirano S, Funakoshi S, Nishi Y, et al. Analysis of factors influencing glucose tolerance in Japanese patients with nonalcoholic fatty liver disease. Diabetol Metab Syndr. 2017;9:65.

13. Stancáková A, Javorský M, Kuulasmaa T, Haffner SM, Kuusisto J, Laakso M. Changes in insulin sensitivity and insulin release in relation to glycemia and glucose tolerance in 6414 Finnish men. Diabetes. 2009;58:1212-21.

14. Retnakaran R, Shen S, Hanley AJ, Vuksan V, Hamilton JK, Zinman B. Hyperbolic relationship between insulin secretion and sensitivity on oral glucose tolerance test. Obesity (Silver Spring). 2008;16:1901-7.

15. Retnakaran R, Qi Y, Goran MI, Hamilton JK. Evaluation of proposed oral disposition index measures in relation to the actual disposition index. Diabet Med. 2009;26:1198-203.

16. Morimoto A, Tatsumi Y, Deura K, Mizuno S, Ohno Y, Miyamatsu N, et al. Impact of impaired insulin secretion and insulin resistance on the incidence of type 2 diabetes mellitus in a Japanese population: the Saku study. Diabetologia. 2013;56:1671-9.

17. Ekelund M, Shaat N, Almgren P, Groop L, Berntorp K, et al. Prediction of postpartum diabetes in women with gestational diabetes mellitus. Diabetologia. 2010;53:452-7.

18. Sung KC, Reaven GM, Kim SH. Utility of homeostasis model assessment of $\beta$-cell function in predicting diabetes in 12,924 healthy Koreans. Diabetes Care. 2010;33:200-2. 
19. Kanazawa I, Yamaguchi T, Yamauchi M, Yamamoto M, Kurioka S, Yano S, et al. Serum undercarboxylated osteocalcin was inversely associated with plasma glucose level and fat mass in type 2 diabetes mellitus. Osteoporos Int. 2011;22:187-94.

20. Takashi Y, Koga M, Matsuzawa Y, Saito J, Omura M, Nishikawa T. Undercarboxylated osteocalcin can predict insulin secretion ability in type 2 diabetes. J Diabetes Investig. 2017;8:471-4.

21. Fukuda-Akita E, Okita K, Okauchi Y, Ryo M, Nakamura T, Funahashi T, et al. Impaired early insulin secretion in Japanese type 2 diabetes with metabolic syndrome. Diabetes Res Clin Pract. 2008;79:482-9.

22. Funakoshi S, Fujimoto S, Hamasaki A, Fujiwara H, Fujita Y, Ikeda K, et al. Analysis of factors influencing pancreatic $\beta$-cell function in Japanese patients with type 2 diabetes: association with body mass index and duration of diabetic exposure. Diabetes Res Clin Pract. 2008;82:353-8.
23. Bilotta FL Arcidiacono B, Messineo S, Greco M, Chiefari E, Britti D, et al. Insulin and osteocalcin: further evidence for a mutual cross-talk. Endocrine. 2018;59:622-32.

24. Shea MK, Gundberg CM, Meigs JB, Dallal GE, Saltzman E, Yoshida M, et al. Gamma-carboxylation of osteocalcin and insulin resistance in older men and women. Am J Clin Nutr. 2009;90:1230-5.

25. Tsugawa N, Shiraki M, Suhara Y, Kamao M, Ozaki R, Tanaka K, et al. Vitamin $\mathrm{K}$ status of healthy Japanese women: age-related vitamin $\mathrm{K}$ requirement for $\gamma$-carboxylation of osteocalcin. Am J Clin Nutr. 2006;83:380-6.

\section{Publisher's Note}

Springer Nature remains neutral with regard to jurisdictional claims in published maps and institutional affiliations.
Ready to submit your research? Choose BMC and benefit from:

- fast, convenient online submission

- thorough peer review by experienced researchers in your field

- rapid publication on acceptance

- support for research data, including large and complex data types

- gold Open Access which fosters wider collaboration and increased citations

- maximum visibility for your research: over $100 \mathrm{M}$ website views per year

At BMC, research is always in progress.

Learn more biomedcentral.com/submissions 